\title{
Las Políticas Públicas Ambientales Como Efectivación del Derecho Humano al Medio Ambiente
}

\author{
Alvaro A. Sánchez Bravo \\ Doctor en Derecho. Profesor de la Facultad de Derecho de \\ la Universidad de Sevilla. España. Profesor V. Programa \\ de Pós-Graduação Stricto Sensu em Ciência Jurídicica. \\ Mestrado e Doutorado. Univali. Presidente de la Asocia- \\ ción Andaluza de Derecho, Medio Ambiente y Desarrollo \\ Sostenible. Coeditor Revista Internacional de Direito \\ Ambiental (Rida).elialva@us.es
}

\section{Resumo}

0 presente estudo trata acerca das políticas públicas ambientais como um meio de efetivação do direito humano ao meio ambiente. Constatou-se a importância transcendental de respeito ao meio ambiente para sobrevivência da própria espécie humana no planeta. 0 modelo de civilização existente tem sido um fracasso no que diz respeito às questões ambientais, sendo necessária uma mudança de paradigma. Assim, impõe o modelo de desenvolvimento sustentável, que, por sua vez, promova 0 desenvolvimento integral dos indivíduos e dos povos para garantir a conservação do nosso ambiente natural permanentemente. A educação ambiental, nesse sentido, é revelada essencial para a transformação social. Por fim, a abordagem leva à conclusão de que são necessárias políticas públicas para a sustentabilidade que satisfaça as necessidades do presente, sem comprometer a capacidade de as gerações futuras satisfazerem as suas próprias necessidades. Diante dessa constatação, vê-se a necessidade de reconhecer legalmente e proteger o meio ambiente como um direito humano, uma vez que não é possível falar em desenvolvimento sustentável sem a universalização e expansão necessárias dos direitos humanos.

Palavras-chave: Políticas públicas. Meio ambiente. Desenvolvimento sustentável. Direitos humanos. 


\title{
The Environmental Public Policies as an Effectivation of the Human Right to Environment
}

\begin{abstract}
The present study is about the environmental public policies as a way of effectivation of the human right to environment. It was found the transcendental importance of the respect to the environment as survival of the human species itself in the planet. The model of civilization that exists has been a failure on what concerns to environmental issues, being necessary a paradigm change. Therefore, the modelo of sustainable development is imposed, that, on the other hand, promotes the integral development of the individuals and people to guarantee the conservation of our natural environment permanently. The environmental education, in this sense, is revealed as essencial for the social transformation. Ultimately, the approach leads to the conclusion that we need public policies about sustainability that satisfies the needs of the presente without compromising the capability of future generations to satisfying their own needs. Before this constatation, it is seen the need of recognizing legally and protecting the environment as a human right, since it is not possible to talk about sustaintable development without the universalization and expansion needed of the human rights.
\end{abstract}

Keywords: Public Policies. Environment. Sustainable Development. Human Rights.

\section{Sumario}

1 Introducción. 2 Medio ambiente y derecho. 3 Delimitación del contenido del derecho al medio ambiente. 4 Políticas públicas ambientales. $5 \mathrm{~A}$ modo de conclusión 


\section{INTRODUCCIÓN}

A nadie escapa hoy en día la transcendental relevancia que tiene respetar nuestro medio ambiente. La amenaza del cambio climático, con sus devastadoras consecuencias, no es más que la constatación del desastre cometido contra nosotros mismos. Nuestro modelo civilizatorio ha sido un fracaso en lo tocante a las cuestiones ambientales, y ha llegado el momento de cambiar de paradigma. Se impone así el modelo de un desarrollo sostenible, que a la vez que promueva el desarrollo integral de los individuos y sus pueblos, garantice la conservación de nuestros entornos naturales de manera permanente.

El Informe Nuestro Futuro Común, de la Comisión Mundial del Medio Ambiente y del Desarrollo (CMMAD, 1998) define el desarrollo sostenible como el desarrollo que satisface las necesidades de la generación presente sin comprometer la capacidad de las generaciones futuras para satisfacer su propias necesidades.

El desarrollo sostenible expresa dos ideas muy claras: el uso racional de los recursos naturales y la protección del ecosistema mundial en las figuras de los ciudadanos (respeto al medio, cambio de hábitos), ciencia (conocimientos y soluciones) y poderes públicos (legislación y cooperación con otros países).${ }^{1} \mathrm{El}$ desarrollo, en consecuencia, debe determinarse como un proceso de cambio común y colectivo hacia el mejoramiento de la calidad de vida de los seres humanos y sus comunidades, mientras que la sostenibilidad es la necesidad de un desarrollo integrado y responsable a nivel social, económico y del medio ambiente, orientado al largo plazo y por lo tanto duradero.

1 Marcos Fernandez, I. El concepto de desarrollo sostenible. Disponible en: <http://www. ecoportal.net/contenido/temas_especiales/desarrollo_sustentable/el_concepto_de_ desarrollo_sostenible>. 
La cuestión de la protección de la naturaleza no se resuelve en una mera cuestión técnica o científica, de expertos, sino que nos involucra a todos los ciudadanos. Porque somos los ciudadanos los depositarios, que no propietarios, de esas riquezas naturales, que sometidas hoy a las leyes del mercado y la globalización sucumben a los intereses particulares de unos pocos en perjuicio de todos. Si destruimos el planeta? A donde iremos?....

Ante esta constatación, cobra carta de naturaleza la necesidad de reconocer y amparar jurídicamente el medio ambiente como un derecho humano. Como puede hablarse de desarrollo sostenible, sin la necesaria universalización y ampliación de los derechos humanos?

\section{MEDIO AMBIENTE Y DERECHO}

Las tensiones constatadas han colocado al Derecho ante la necesidad de considerar esta nueva demanda social, y sus respuesta no se ha hecho esperar. La más relevante iniciativa es la que concibe el medio ambiente como un derecho humano.

Como señala Pérez Luño, los derechos humanos aparece como un conjunto de facultades e instituciones que, en cada momento histórico, concretan las exigencias de la dignidad, la libertad y la igualdad humanas, las cuales deben ser reconocidas positivamente por los ordenamientos jurídicos a nivel nacional e internacional. ${ }^{2}$ Por tanto, en esta hora presente, ¿que más exigencia de dignidad, libertad e igualdad se concentra en la defensa de nuestro medio ambiente?

2 Pérez Luño, A. E. Derechos humanos, Estado de Derecho y constitución. 9. ed. Madrid: Tecnos, 2005. 
Los derechos cumplen una doble función: delimitar y garantizar las libertades existentes y señalar el objetivo último a alcanzar. En la cuestión que nos ocupa, debemos hablar de una inversión del segundo objetivo: se trata precisamente no de alcanzar el objetivo que la ciencia nos enseña a diario: la destrucción del planeta. El reconocimiento y protección del medio ambiente como derecho humano debe ser el instrumento a través del cual evitemos ( si es posible) o atenuemos esa destrucción.

Desde la aparición del hombre sobre el planeta, su relación ha sido necesariamente con la naturaleza, en su doble dimensión de ser biológico, y como unto de referencia para su supervivencia y transformación. Esto que parece obvio, no obstante no es asumido correctamente por algunas tendencias del ecologismo, que obvian la necesaria consideración antrópica de la percepción de la naturaleza, abogando por paraísos ideales inimaginables.

Naturaleza y ser humano, y posteriormente naturaleza y sociedad, son las dimensiones de una realidad inseparable, cuyo equilibrio dependerá de los sistemas de producción, de los avances tecnológicos y científicos y de la estructura y organización sociales. ${ }^{3}$

El proceso globalizador en lo económico y lo social ha provocado una conciencia social más sensible hacia las cuestiones ambientales que ha propiciado una presión hacia nuevas regulaciones tendentes a la incorporación de medidas de protección y conservación de la naturaleza, a remediar los daños ambientales y a gestionar más eficazmente los impactos de los procesos productivos.

3 Ibid. 
El camino hacia el reconocimiento del medio ambiente como derecho humano es un proceso apenas iniciado, y está lleno de contradicciones, aunque avanza lentamente hacia la positivación en los ordenamientos jurídicos nacionales e internacionales ${ }^{4}$.

Ahora bien, ¿cuáles son las elementos a considerar para la correcta articulación del medio ambiente como derecho humano?

\section{DELIMITACIÓN DEL CONTENIDO DEL DERECHO AL MEDIO AMBIENTE.}

La regulación del medio ambiente presenta numerosas aristas, y es objeto de una consideración diversa en el derecho comparado. Para contribuir al esclarecimiento de que sea el derecho al medio ambiente y que status le corresponde, consideramos imprescindible, detenernos en los tres elementos que, correctamente articulados, permitirán su máximo reconocimiento y eficacia.

1. Delimitación del concepto de medio ambiente. La denominación medio ambiente es una expresión poco precisa. Así puede hablarse de un medio ambiente natural, integrado por todos los elementos que sirven de soporte a la vida, y por los sistemas producto de la interacción de los seres vivos con el medio natural. Pero también cabe hablar de un medio ambiente construido por los hombre desde el inicio de las sociedades para satisfacer el cada vez más amplio elenco de necesidades.

Consideramos adecuado, desde el punto de vista jurídico, la opción por una visión amplia e integradora del medio ambiente, como el conjunto de condiciones externas que conforman el contexto de la vida humana. ${ }^{5}$

${ }_{4}$ Loperena Rota, D. Los derechos al medio ambiente adecuado y su protección. Disponible en: <http://www.cica.es/aliens/gimadus/loperena.html>.

5 Perez Luno, A. E. Derechos Humanos... cit. 
La respuesta jurídica debe producirse ante la constatación de que ese conjunto de condiciones pueden alterarse por causas antropogénicas poniendo en peligro la vida sobre el planeta, y especialmente la nuestra.

2. Medio Ambiente y Calidad de Vida. Como venimos señalando, su concreta vinculación con la existencia humana, el desarrollo y la satisfacción de las necesidades, es lo que hace relevante al medio ambiente como derecho para ser incluido en el catálogo de los derechos fundamentales. Ahora bien, una precisión debe ser considerada: como señala Pérez Luño, "las técnicas de positivación de los derechos fundamentales requieren que éstos se refieran a situaciones bien determinadas en cuanto a su objeto y titularidad. Ahora bien, resulta difícil establecer con precisión el conjunto de facultades constitutivas del derecho al medio ambiente". Por eso, para evitar caer en una cuestión meramente simplista el reconocimiento de un derecho al ambiente no se cierra en la simple opción por la aparición de un derecho nuevo o específico, dado la pluralidad de situaciones a las que alude. ${ }^{6}$

Pero, a mayor abundamiento, si asumimos que los derechos fundamentales presentan una inmediata conexión con los valores que la sociedad considera imprescindible para su propia existencia, la constatación, en demasiadas ocasiones, de un medio ambiente arrasado nos lleva a que éste no sea sólo un mero objeto de regulación normativa y pase a convertirse en un valor imprescindible para el desarrollo de los seres humanos. Surge así el concepto de "calidad de vida”. Como señala el profesor de la Hispalense, “esta noción refleja una réplica a la idea puramente cuantitativa del bienestar y postula un desarrollo cualitativo y equilibrado, en armonía con la naturaleza; y que sustituye el consumismo por la satisfacción de necesi-

6 Perez Luno, A. E. Derechos Humanos... cit. 
dades humanas básicas de acuerdo con el principio de solidaridad". ${ }^{7}$ No se trata, por tanto de proteger cualquier medio ambiente, sino que éste debe ser el adecuado para garantizar nuestra calidad de vida.

La apuesta por la opción medio ambiente-calidad de vida otorga a su regulación positiva en los ordenamientos más fuerza, al imponer un mandato al resto del ordenamiento jurídico, pero también encomienda a los poderes públicos la remoción de los obstáculos que impidan su realización, y generen desigualdades y abusos. Sobre esta cuestión volveremos al abordar las políticas públicas ambientales.

3. Inclusión en el "núcleo duro constitucional". Frente a su incorporación bajos formulas de principios programáticos 0 , como hace nuestra constitución española, en los "principios rectores de la política social y económica”, la inclusión del derecho al medio ambiente, en su conexión con la calidad de vida, debe integrarse en aquel conjunto de derechos, "núcleo duro" que refuerzan su protección y garantía por los ordenamientos jurídicos especialmente los constitucionales. Y ello, porque se ven reforzados desde tres planos:

a) Procedimiento agravado de reforma constitucional, que impide que las simples mayorías parlamentarias coyunturales puedan modificar a su albur los derechos de los ciudadanos, e atención a intereses partidistas o coyunturales.

b) Los derechos vinculan no sólo a los poderes públicos , sino también a los ciudadanos, pudiendo ser alegados ante o aplicados por los tribunales de justicia.

c) Reserva de Ley. Los derechos fundamentales deben ser regulados por instrumentos normativos que garanticen la integridad del significado y función de los mismos. Pero no bastará cualquier desarrollo

7 Ibid. 
legislativo, sino que debe garantizar el "contenido esencial" de los derechos evitando que su regulación sea una formula encubierta para impedir su aplicación, la dificulte o la despoje de sus elementos de garantía. ${ }^{8}$

\section{POLÍTICAS PÚBLICAS AMBIENTALES.}

Como venimos indicando, la consideración del medio ambiente, en su conexión con la calidad de vida, supone la vinculación de los poderes públicos y de los particulares en la defensa de los valores medioambientales.

Siguiendo lo señalado por Pérez Luño, son tres las funciones asignadas a los poderes públicos:

1. Función preventiva. Los poderes públicos deben velar por una utilización racional de los recursos naturales, con el objetivo de proteger la calidad de vida.

2. Función restauradora. Los poderes públicos no sólo deben tutelar el medio ambiente, sino que debe extenderse a la reparación de los posibles daños y agresiones.

3. Función promocional. Los poderes públicos deben contribuir a la mejora del medio ambiente, incorporando políticas activas de mejora y recuperación ambiental. ${ }^{9}$

Ahora bien, la cuestión está en determinar cuales son los mecanismos para que esas funciones se cumplan de manera efectiva, y no suceda, como acaece con demasiada frecuencia, que queda en mera retórica o simple declaración de intenciones políticas sin traslado a los ciudadanos.

8 Perez Luño, A. E. Los derechos fundamentales. 2. ed. Madrid: Tecnos, 1986.

9 Perez Luño, A. E. Derechos humanos... cit. 
El Objetivo Séptimo del Desarrollo del Milenio, Garantizar la sostenibilidad ambiental, establece entre sus metas incorporar los principios del desarrollo sostenible en las politicas y los programas nacionales e invertir la pérdida de recursos ambientales. ${ }^{10}$

Como indicamos al principio de nuestra reflexión, el nuevo paradigma del desarrollo sostenible, incorpora no sólo la defensa de los valores ambientales, sino que uno de los elementos claves e imprescindibles para su consecución es el desarrollo de los individuos y las sociedades en las que se integra. Es por ello que las políticas públicas tiene que partir de la premisa de que muchos problemas ambientales tienen su foco en la pobreza.

La reducción de la pobreza puede devenir un elemento de primer orden para luchar contra la degradación ambiental, pues no olvidemos que ésta muestra su rostro más dramático con los pobres.

\footnotetext{
${ }^{10}$ Disponible en: <http://www.undp.org/spanish/mdg >. La Declaración del Milenio fue aprobada en la Cumbre del Milenio, celebrada del 6 al 8 de septiembre de 2000 en Nueva York, con la participación de 191 países (siendo 189 Estados Miembros en ese entonces), incluyendo a 147 jefes de Estado y de gobierno. Los objetivos acordados en esta Cumbre se conocen ahora como los Objetivos de Desarrollo del Milenio (los ODM) y proporcionan un marco para que todo el sistema de la ONU trabaje coherentemente y en conjunto hacia un fin común. El Grupo de las Naciones Unidas para el Desarrollo ayudará a asegurar que los ODM permanezcan al centro de estos esfuerzos.

Los objetivos de desarrollo del milenio son 8: 1)la erradicación del hambre y la pobreza, 2) la educación primaria universal, 3) la igualdad de género, 4) reducir la mortalidad infantil, 5)mejorar la salud materna, 6) la detención del avance del VIH/SIDA, paludismo y tuberculosis, 7) garantizar la sostenibilidad del medio ambiente y 8) fomentar una asociación mundial para el desarrollo.
} 
Es por ello, que se requiere de políticas que hagan hincapié en la complementariedad entre el desarrollo sostenible y la gestión ambiental. Garantizar la sostenibilidad ambiental permitirá, cumplir con el resto de Objetivos de Desarrollo del Milenio. ${ }^{11}$

11 "La adopción de los objetivos de desarrollo del Milenio, extraídos de la Declaración del Milenio, fue un acontecimiento fundamental en la historia de las Naciones Unidas. Constituyó una promesa sin precedentes de los dirigentes mundiales de abordar, de una sola vez, la paz, la seguridad, el desarrollo, los derechos humanos y las libertades fundamentales. Como dije en mi informe de marzo de 2005, titulado "Un concepto más amplio de la libertad: desarrollo, seguridad y derechos humanos para todos", que complementa el presente informe: "no tendremos desarrollo sin seguridad, no tendremos seguridad sin desarrollo y no tendremos ninguna de las dos cosas si no se respetan los derechos humanos. A menos que se promuevan todas esas causas, ninguna de ellas podrá triunfar". Los ocho objetivos de desarrollo del Milenio van desde reducir a la mitad la pobreza extrema hasta detener la propagación del VIH/SIDA y lograr la educación primaria universal, todo ello, a más tardar en 2015” Kofi A Annan, en Objetivos del desarrollo del Milenio. Informe 2005, ONU, Disponible en: <http://millenniumindicators.un.org/unsd/ mi/pdf/MDG\%20BOOK_SP_new.pdf>. 


\section{Por qué conseguir el Objetivo ambiental es tan importante para los restantes Objetivos}

Objetivos

Relación con el medio ambiente

1. Erradicar la pobreza extrema y el hambre

2. Lograr la educación primaria universal

3. Promover la igualdad entre los géneros y la autonomía de la mujer

4. Reducir la mortalidad infantil

5. Mejorar la salud materna

6. Combatir las principales enfermedades

8. Fomentar una asociación mundial para el desarrollo
El sustento y la seguridad alimentaria de los pobres dependen a menudo de los bienes y servicios del ecosistema. Los pobres suelen tener pocos derechos sobre los recursos ambientales y un acceso inadecuado a los mercados, a la toma de decisiones y a la información ambiental, lo que limita su capacidad de proteger el medio ambiente y mejorar su sustento y bienestar. La falta de acceso a los servicios energéticos también limita las oportunidades de producción, especialmente en las zonas rurales.

El tiempo dedicado a ir a buscar agua y leña reduce el tiempo disponible para la educación. Además, la falta de energia, agua y servicios sanitarios en las zonas rurales desanima a los maestros cualificados a trabajar en los pueblos pobres.

Generalmente, son las mujeres y las niñas las que cargan con la recogida de agua y combustible, lo que reduce el tiempo y las oportunidades de que disponen para la formación, alfabetización y realización de actividades generadoras de beneficios. A menudo, las mujeres tienen menos derechos que los hombres y carecen de tenencia segura de la tierra y otros recursos naturales, lo que limita sus oportunidades y capacidad para acceder a otros bienes de producción.

Las enfermedades ligadas a la suciedad del agua y a una higiene inadecuada (como la diarrea) y las infecciones respiratorias relacionadas con la contaminación son las principales responsables de la mortalidad de los niños menores de cinco años. La falta de combustible para hervir el agua tambien contribuye a la existencia de enfermedades transmitidas a través del agua que se pueden prevenir.

Respirar aire contaminado en el interior de las viviendas y transportar grandes cargas de agua y leña perjudica la salud de las mujeres, que como consecuencia de ello pueden tener más problemas y complicaciones durante el embarazo. Sin energla para iluminación ni refrigeración y sin condiciones higiénicas adecuadas, la atención médica es muy precaria, especialmente en las zonas rurales.

Hasta el $20 \%$ de las enfermedades que afectan a los paises en desarrollo puede deberse a factores de riesgo ambiental (tal y como sucede con la malaria y las infecciones parasitarias). Las medidas preventivas para reducir tales peligros son tan importantes como el tratamiento en si y a menudo son más rentables. Las nuevas medicinas obtenidas de la biodiversidad son una promesa para luchar contra las principales enfermedades.

Muchos problemas ambientales mundiales (el cambio climático, la pérdida de la diversidad de especies, el agotamiento de las reservas pesqueras mundiales) únicamente pueden solucionarse mediante asociaciones entre paises ricos y pobres. Asimismo, las inversiones depredadoras de recursos naturales pueden hacer aumentar la sobreexplotación de los activos medioambientales en los paises pobres. 
Ahora bien, las actuaciones políticas deben tener presentes los diferentes entornos naturales, las variadas y difusas causas de la degradación ambiental así como los estrechos lazos entre pobreza y medio ambiente. Como señalaba el Informe sobre Desarrollo Humano 2003, ${ }^{12}$ las políticas ambientales deberían descansar sobre seis grandes principios:

1. Reforzamiento de las instituciones y de la gobernabilidad.

Una parte importante de los problemas ambientales tienen su razón de ser en fallos institucionales y en la debilidad de los gobiernos. En lo tocante a la gestión ambiental se indican tres disfuncionalidades: el mantenimiento de derechos de propiedad y de uso y gestión inadecuado de los recursos naturales, la falta de información a los ciudadanos, y la escasa implantación y supervisión del cumplimiento de la normativa ambiental.

Ello viene motivado en gran parte por la adopción de medidas políticas al margen, cuando no en contra, de las comunidades y ciudadanos a las que van dirigidas. Es por ello que debe incrementarse la participación ciudadana y la descentralización, pero con el horizonte de crear capacidad de gestión de los recursos y de intervenir en la planificación y elaboración de las políticas.

Igualmente, en aquellos supuestos, en los que el desarrollo de instituciones formalizadas pueda ser dificultosa para los Estados, debe poder recurrirse a mecanismos informales de regulación, basados en la intervención de las comunidades afectadas, y en su posibilidad de solicitar responsabilidades por la gestión.

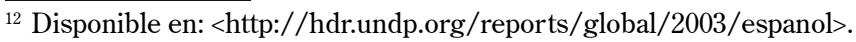


La mejora de la legislación y de su cumplimiento, no debe entenderse como una desregulación, sino como una mejora de las políticas y sus objetivos en beneficio de todos los interesados. Además supondrá que las políticas en los diferentes ámbitos se complementen y sean coherentes entre sí y determinen las aportaciones mutuas. ${ }^{13}$

2. La sostenibilidad ambiental como política horizontal.

La mayor parte de las políticas afectan al medio ambiente, pero en numerosas ocasiones no se tiene en cuenta en la adopción de decisiones.

Los objetivos medioambientales deben tenerse en cuenta desde las primeras fases del proceso de elaboración de todas las políticas, de la agrícola a la económica. Para cumplir este objetivo de manera satisfactoria, es preciso conocer mejor el origen de los problemas ecológicos y cómo interactúan entre sí, y disponer de una sólida base científica. Es necesario, además, medir los progresos realizados. La aplicación de metas e indicadores va a ayudarnos a nosotros y a los ciudadanos a evaluar los resultados conseguidos. Nos permitirá, asimismo, saber enseguida si nuestras políticas están siendo suficientemente eficaces. ${ }^{14}$

3. Mejora de la competitividad y supresión de las ayudas perjudiciales al medio ambiente.

La separación entre beneficios privados y públicos de la actividad económica debe superarse. En este campo, donde la sociedad asume los costes ambientales de numerosas actividades, deben establecerse criterios correctores. La fiscalidad ambiental puede ser un instrumento adecuado

${ }^{13}$ Documento de Trabajo de la Comisión. La mejora de la legislación y las estrategias temáticas en el ámbito del medio ambiente, COM (2005) 466 final / SEC (2005) 1197, Bruselas, 28.09.05.

${ }^{14}$ Medio ambiente 2010: El futuro está en nuestras manos. Programa de Acción de la Comunidad Europea en materia de Medio Ambiente. Oficina de Publicaciones Oficiales de la Comunidades Europeas, Luxemburgo, 2001. 
en este ámbito. Así en Andalucía, la Ley 18/2003, de 29 de diciembre, señala en su Exposición de Motivos, que, como medios complementarios para coadyuvar a la protección y defensa del medio ambiente, las medidas en materia de fiscalidad ecológica incluyen un abanico de figuras impositivas con la finalidad de estimular e incentivar comportamientos más respetuosos con el entorno natural. Asimismo, la recaudación que proporciona esta clase de mecanismos compensará el impacto en los recursos naturales que originan las conductas humanas, contribuyendo, de este modo, a sufragar las acciones incluidas en las políticas medioambientales concretas, ya sean proyectos, ayudas o fondos destinados a situaciones de emergencia.

Por su parte, la experiencia brasileña, con el Imposto de Mercadorias e Serviços o ICMS-E, muestra bien a las claras lo relevante que puede ser la utilización de impuestos ecológicos como mecanismos de fomento de la igualdad y defensa del medio ambiente. ${ }^{15}$

Otro elemento relevante vendrá constituido por la inclusión de los costos ambientales en los precios de mercado de los productos y servicios ofertados a los ciudadanos. Especialmente interesante es lo señalado por

${ }^{15}$ En 1992, en la mayor parte de los estados brasileños se adoptó un impuesto ecológico sobre el valor añadido (Imposto sobre Circulação de Mercadorias e Serviços, o ICMS-E). El ICMS, impuesto sobre bienes, servicios, energía y comunicaciones, es la mayor fuente de ingresos de Brasil. Una cuarta parte de la recaudación se destina a los municipios, repartiéndose entre ellos según diferentes indicadores de desempeño ambiental. Los estados de Paraná y Minas Gerais, por ejemplo, distribuyen la recaudación según la proporción de zonas protegidas en cada municipio, ponderado por un factor de conservación relacionado con la protección de cada zona. El ICMS-E pretendía compensar a los municipios con grandes zonas de conservación por la pérdida de ingresos que esto conlleva. La recaudación procedente de este impuesto a menudo se emplea para conservar los parques y reservas, incluyendo la compra de herramientas y los salarios del personal. En algunos estados, parece que este impuesto ha hecho aumentar de forma importante el número y tamaño de las zonas protegidas. En Paraná, las zonas de conservación crecieron en más de 1 millón de hectáreas entre 1991 y 2000, lo que supone un aumento del 165\%. Entre 1995 y 2000, en Minas Gerais crecieron también en más de 1 millón de hectáreas (un aumento del 62\%). Disponible en: <http://hdr.undp.org/reports/global/2003/espanol>. 
la Directiva Marco de Aguas en la Unión Europea, ${ }^{16}$ cuando señala que "el principio de recuperación de los costes de los servicios relacionados con el agua, incluidos los costes medioambientales y los relativos a los recursos asociados a los daños o a los efectos adversos sobre el medio acuático, deben tenerse en cuenta, en particular, en virtud del principio de que quien contamina paga”.

Cuestión a considerar también sería la relativa a la consideración de los impactos de las actividades económicas en los ecosistemas naturales, discriminando entre aquellas actividades sostenibles, y aquellas que suponen la merma de nuestros recursos. Se trata, pues, de crear una "contabilidad ambiental” que permita justipreciar adecuadamente los costes de los proceso económicos y permita planificar y dar más relevancia a los atentados ambientales. Obligación que debe extenderse a las propias administraciones públicas. El objetivo es introducir criterios "verdes" en la contratación pública asumiendo la responsabilidad de reducir al mínimo los impactos ambientales en la compra de bienes y servicios y presentar una demanda de éstos que pueda ser detectada por los productores y proveedores de la administración, a fin de que encuentren interesante producir y distribuir nuevas alternativas sostenibles a los productos y servicios tradicionales. ${ }^{17}$ La elaboración de manuales para ayudar a integrar criterios ambientales en los procedimientos de compras públicas constituye una iniciativa exitosa, que podría incorporarse a otras legislaciones. ${ }^{18}$

${ }_{16}$ Directiva 2000/60/CE del Parlamento Europeo y del Consejo, de 23 de octubre de 2000, por la que se establece un marco comunitario de actuación en el ámbito de la política de aguas, Doce L 327. 22.12.2000.

17 “Compras verdes en la contratación pública. Dar ejemplo: ¡Esa es la clave!”. En: Ambienta. Revista del Ministerio de Medio Ambiente, n. 52, febrero 2006, p. 13.

${ }^{18}$ Comision Europea, ;Compras Ecológicas! Manual sobre la contratación pública ecológica, Oficina de Publicaciones Oficiales de las Comunidades Europeas, Luxemburgo, 2005. Disponible en: <http://ec.europa.eu/environment/gpp/pdf/buying_green_handbook_ es.pdf>. 
Desde el punto de vista de los productores, cobra carta de naturaleza la apuesta por un modelo de responsabilidad social corporativa social y ambiental, que permita la puesta en marcha de políticas consensuadas entre la administración y la industria, con el común objetivo de garantizar la defensa de nuestros valores ambientales, garantizando a la vez el desarrollo económico y social de los pueblos. ${ }^{19}$

4. Refuerzo de la cooperación internacional en la gestión ambiental.

Los atentados ambientales rara vez se detienen en las fronteras, no conociendo ni de legislaciones ni de jurisdicciones. Sucesos como Chernobil o la destrucción del Mar Aral, son una buena muestra de la triste globalización de los daños ambientales. La cooperación internacional se revela imprescindible para luchar contra esta lacra. Los Estados deben cooperar trabajar en común, pues las acciones de unos perjudican y/o benefician a los otros.

Debe mejorarse la cooperación internacional, estableciendo metas y objetivos adecuados a las necesidades sociales y ambientales reales, al margen de estrategias de poder o de zonas de influencia ( siempre interesadas).

Los temas relacionados con el medio ambiente deberían abordarse en los diálogos periódicos con los terceros países, para definir una percepción común de las prioridades ambientales que conviene aplicar. Con los países industrializados, los temas abordados deberían ser los que son objeto de convenios internacionales o de protocolos, o los que se debaten

${ }^{19}$ Comunicación de la Comisión al Parlamento Europeo, al Consejo y al Comité Económico y Social Europeo. Poner en práctica la asociación para el crecimiento y el empleo: hacer de Europa un polo de excelencia de la responsabilidad social de las empresas. COM (2006) 136 final. Bruselas. 22.3.2006. 
en los foros multilaterales. Con los países en desarrollo, las preocupaciones ambientales deberían integrarse en los instrumentos y programas de cooperación.

En los ámbitos de actuación horizontales, los objetivos clave son los siguientes:

- apoyo a las mejoras en el ámbito de los derechos humanos, de la democratización y de la gobernanza, que también contribuyan a fines ambientales;

- consideración del medio ambiente en las actividades de prevención de conflictos, para reducir las tensiones relacionadas con el acceso a los recursos naturales y a su utilización;

- fomento de la dimensión ambiental en todos los ámbitos de reconstrucción tras un conflicto.

Las negociaciones y las relaciones comerciales también deberán tener en cuenta las preocupaciones ambientales y deberán aclararse las relaciones entre el sistema comercial internacional y los acuerdos sobre medio ambiente. Deberá fomentarse la responsabilidad social de las empresas a escala internacional, así como la asistencia técnica, las transferencias de tecnologías limpias y la realización de estudios de impacto.

Deberá mejorarse el marco institucional internacional, especialmente mediante la integración sistemática de los temas de índole ambiental en las preocupaciones de los foros internacionales. Además, debe garantizarse una mayor coherencia entre los debates bilaterales y multilaterales, así como entre las distintas organizaciones internacionales. $^{20}$

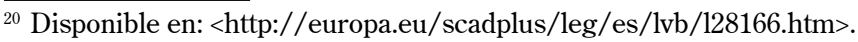


5. Mejora de la tecnología y los conocimientos ambientales.

Es preciso aumentar las inversiones destinadas a investigación y desarrollo, teniendo presente las dificultades que afrontan los países pobres a la hora de implementar tecnologías ambientales adecuadas.

Los conocimientos sobre los ecosistemas naturales son ingentes, pero aún queda mucho por descubrir. Se debe seguir avanzando en la cooperación con los comités científicos internacionales aportando recursos e información. Además, esto permitirá aprovechar el valor añadido de sus pesquisas, pues los responsables políticos necesitan, a la hora de elaborar su previsiones, disponer de datos fiables y adecuados sobre los problemas ambientales generados por nuestras actividades.

El trabajo del IPCC es un ejemplo a seguir en este campo. ${ }^{21}$ 6. Apuesta por la conservación.

La creación de zonas protegidas constituye una opción imprescindible para conservar la diversidad de especies y ecosistemas.

La conservación es parte fundamental de la estrategia de gestión sostenible del territorio y sus recursos. Por ello, los Espacios Naturales Protegidos no son entidades aisladas del resto del territorio sino que han de formar parte de un sistema de conservación en el que se contempla una gradación continua desde áreas naturales, prácticamente inalteradas, hasta la ciudad, donde los procesos naturales son controlados al máximo.22

Ahora bien, los procesos de conservación deben tener presentes a los pobladores de las áreas a proteger. Ellos generalmente han sido y so los depositarios de los conocimientos y de la correcta gestión de los ecosistemas que ha permitido que lleguen hasta nuestros días. La protección

${ }^{21}$ Disponible en: $<\mathrm{http}: / /$ www.ipcc.ch $>$.

${ }^{22}$ Disponible en: <http://www.unescoeh.org/ext/manual/html/espaciosprotegidos2.html $>$. 
de determinadas zonas no puede ser un cheque en blanco para que se borre de un plumazo la historia de un pueblo. Donde la actividad humana sea sostenible y contribuya al mantenimiento del ecosistema debe mantenerse, aprovechar los conocimientos de la población autóctona para exportarlos a otras áreas, y ampliar la protección. Donde así no sea, si tendrá que intervenir la administración apara evitar el efecto contrario indeseado: que la explotación abusiva de unos, y su beneficio, nos perjudique a todos.

\section{A MODO DE CONCLUSIÓN}

La defensa del medio ambiente como derecho fundamental, constituye hoy un reto en los sistemas constitucionales. El otorgamiento del máximo nivel de consideración jurídica y social, que duda cabe, contribuirá a un aumento de su protección, rodeándolo de instrumentos de garantía que aseguren su eficacia. Las políticas públicas juegan un rol fundamental una vez que el reconocimiento constitucional y jurídico se ha producido. Como sabemos, una cosa es legislar, y otra cumplir y llevar a buen puerto lo legislado. Además el desarrollo de los programas jurídicos y políticos requieren de inversiones que la Administración debe tener presentes, para que aquellos no queden en mera retórica. Las políticas públicas en materia ambiental sieguen siendo las grandes olvidadas. Sectores como la agricultura, el turismo, la industria y otros, de gran capacidad de generar riqueza y empleo, pero con graves impactos ambientales, siguen primando sobre las necesaria sostenibilidad social y ambiental. Quienes así actúan olvidan algo obvio: cuando el planeta llegue a un nivel de degradación tal que sea irreversible, nada más tendrá valor, pues ni siquiera la supervivencia estará asegurada. No se trata de ser catastrofistas, sino responsables. Es el momento en que los datos científicos nos muestran bien a las claras hacia donde caminamos. Es el momento de actuar, del compromiso solidario, de la defensa de nuestra propia existencia.... 
Por último, lo más importante, los ciudadanos. Las políticas públicas en pro de la sostenibilidad serán inoperantes sino son asumidas por los ciudadanos en una doble vertiente: participación en la adopción de las decisiones a tomar y compromiso en la implantación de las medidas a desarrollar. Debemos estar atentos y preparados para reivindicar nuestros derechos, y hacerlos cumplir, pero también seremos responsables de nuestros propios actos, y sólo podremos exigir aquellos que estamos dispuestos a cumplir.

Los ciudadanos debemos involucrarnos en una nueva cultura de la sostenibilidad, trasladando a nuestros quehaceres cotidianos la sensibilidad y el compromiso con las cuestiones ambientales.

La educación ambiental, en su sentido más amplia, se revela como imprescindible para la generación de esa nueva sociedad que pretendemos crear y en la que sin renunciar al progreso y al desarrollo seamos más solidarios con el resto de personas y cuidemos del planeta que aún nos sustenta, ampara y protege.

Como señaló Rousseau, "el progreso civilizatorio empuja a los hombres al envilecimiento si no persigue el objetivo de restablecer con sus medios la naturaleza, para, albergados por ella, armónicamente reconciliados con ella, llevar una vida de concordia y de sentido común siempre presente".

Recebido em: 12/9/2013

Aceito em: 28/9/2013 